\title{
PROCESO PARA AUTOEVALUAR LA UNIVERSIDAD TÉCNICA ESTATAL DE QUEVEDO
}

\author{
${ }^{\bullet}$ Román Estuardo Soria Velasco ${ }^{1,2}$ \\ ${ }^{1}$ Unidad de Evaluación Institucional CEI, Universidad Técnica Estatal de Quevedo, $\mathrm{km} 1$ 1/2 vía \\ Quevedo-Santo Domingo de los Tsáchilas, C. P. 73. Quevedo, Los Ríos, Ecuador. ${ }^{\triangle}$ rsoria@uteq.edu.ec \\ ${ }^{2}$ Facultad de Ciencias Pecuarias, Universidad Técnica Estatal de Quevedo, $\mathrm{km} 7$ vía a El Empalme \\ Mocache, Los Ríos, Ecuador
}

\begin{abstract}
RESUMEN
E n este artículo se expone el proceso adoptado por la Universidad Técnica Estatal de Quevedo, para cumplir con la Autoevaluación Institucional previa a la Evaluación Externa, en el camino de su acreditación, según los requerimientos del Consejo Nacional de Evaluación y Acreditación Ecuatoriano (CONEA). Se describen los procedimientos en detalle y sus consecuencias sobre el cumplimiento de estándares en el proceso de Autoevaluación y en el proceso de Evaluación Externa, cumplidos por la universidad. Los resultados logrados evidencian la importancia del proceso en la construcción de la calidad universitaria, con la participación de todos los actores universitarios.
\end{abstract}

Palabras claves: Autoevaluación, Evidencias, Informante, Estándares, Indicador, Planes de mejora.

\begin{abstract}
This article describes the process adopted by UTEQ in order to accomplish the Institutional Self-Assessment previous to the external review on its way to be acredited according to CONEA requirements. Procedures are outlined in detail and its impacts on the fulfillment of standards in the process of Self-Assessment and the External Review processes performed by the university. The results obtained shown the importance of this process in the built of the university's quality, with the participation of all its members.
\end{abstract}

Key words: Self-Assessment, Evidence, Informant, Standards, indicator, improvement plans.

\section{INTRODUCCIÓN}

$\mathrm{L}$ a Constitución Ecuatoriana 1988, la Ley Orgánica de Educación Superior 2000 y el Reglamento General del Sistema Nacional de Autoevaluación y Acreditación, vigentes en el año 2008, establecen la obligatoriedad que tienen la Instituciones de Educación Superior de llevar adelante procesos de Autoevaluación con fines de Acreditación. El Consejo Nacional de Evaluación y Acreditación estableció el Modelo de Autoevaluación y la Guía para Autoevaluación (CONEA, 2003a)

Conceptualmente Evaluar es formular juicios de valor y la Autoevaluación es un tipo de evaluación llevada a cabo por el propio actor sobre sus actuaciones (UP, 2008). Estos juicios de valor tienen tres características fundamentales:

- $\quad$ Son estimaciones de certeza, tanto en el sentido de aproximación, como en el sentido de aprecio por parte del evaluador y por lo tanto exigen Información, Evidencias del Objeto a Evaluar

- $\quad$ Son comparativos, relativos a un Estándar o Criterio previamente establecido

- $\quad$ Son de utilidad, y por lo tanto tienen un propósito, definido por el evaluador.

El Objetivo General del Proyecto de Autoeva-

Recibido: Enero, 2009. Aceptado: Febrero, 2009.

Publicado como NOTA TÉCNICA en Ciencia y Tecnología 2(2): 7-13. 2009 luación de la UTEQ fue: Realizar la Autoevaluación con Fines de Acreditación Institucional mediante procesos de mejoramiento de la calidad de la Educación Superior (CEI, 2006). Es decir el propósito fue mejorar la calidad, esto es, mejorar el trabajo individual de cada integrante de la universidad, integrantes identificados como informantes calificados del proceso. Por lo tanto, cada uno de los informantes calificados debió evaluar el trabajo o gestión que estuvo realizando, para mejorar su desempeño y participación en la construcción de la calidad institucional.

La calidad institucional estuvo esquematizada en las 21 Características, 110 Estándares y 118 preguntas orientadoras de los Principios y Características de Calidad de la Universidad Ecuatoriana (CONEA, 2003b) En consecuencia con la conceptualización ya señalada; estos son los criterios a utilizar para formular los juicios de valor.

La guía del CONEA propuso 179 indicadores que señalan las evidencias documentadas o información pertinente, requerida para formular los juicios de valor. Con esta información los juicios de valor o estimaciones de certeza, fueron menos alejadas de la realidad, y más apreciadas por cada uno de los informantes calificados o evaluadores primarios y además, 
por los evaluadores externos que revisaron el proceso.

Con estos antecedentes, fue necesario diseñar un proceso que cumpla con los requisitos teóricos anteriormente señalados y promueva la cultura de la calidad entre toda la comunidad universitaria. En el año 2008 la UTEQ adoptó un proceso donde, cada informante calificado debía comprender la información o conocimiento que proporciona cada uno de los indicadores que por su trabajo le correspondía informar, mediante un análisis de fortalezas y debilidades derivadas de las evidencias disponibles al momento de evaluar. A continuación se compara las evidencias con los requerimientos descritos en los estándares de calidad y preguntas orientadoras, y después del análisis se formuló el juicio de valor para el indicador analizado, juicio de valor que fue de utilidad para el trabajo diario (Figura 1). En esta nota técnica se analiza si el procedimiento influyó positivamente en la mejora de la calidad institucional definida por el CONEA.

\section{Procedimientos}

E 1 proceso institucional se desarrolló en tres etapas (Soria, 2008). La primera, dedicada a recolectar información (Datos, Hechos) mediante entrevistas, conducidas por los miembros de la Comisión de Evaluación Interna (CEI); donde cada uno de los informantes califi- cados del medio interno debía recopilar la información pertinente al indicador de la guía del CONEA, que le correspondía evaluar, en función de sus responsabilidades y actividades. Para su fiel cumplimiento, cada uno firmó un acta de compromiso. En caso de no existir evidencia para emitir el juicio de valor o la evidencia indicaba incumplimiento del indicador, el mismo informante debía diseñar y, de ser el caso, conducir acciones o planes de mejora aplicables para superar la deficiencia.

La segunda etapa consistió en el análisis de las evidencias. Se inició con talleres de trabajo donde participaron investigadores, empleados, y estudiantes para analizar los aspectos más críticos y poco comprendidos, tanto en el medio interno como en el externo. Aquí los datos, hechos, y/o información recibida por los asistentes a los talleres, entregada por los especialistas en el tema, fue interpretada y explicada, con la ayuda de un análisis FODA, a fin de transformarla en conocimiento que permita comprender y evaluar el tema señalado por el indicador en análisis.

Para el análisis de las evidencias, con la presentación de los referentes para evaluar a todos los funcionarios universitarios, fue necesario realizar talleres de trabajo, para explicar y orientar el procedimiento a seguir, además sirvieron para comprender la importancia de cumplir con los estándares, y cómo cada uno de los

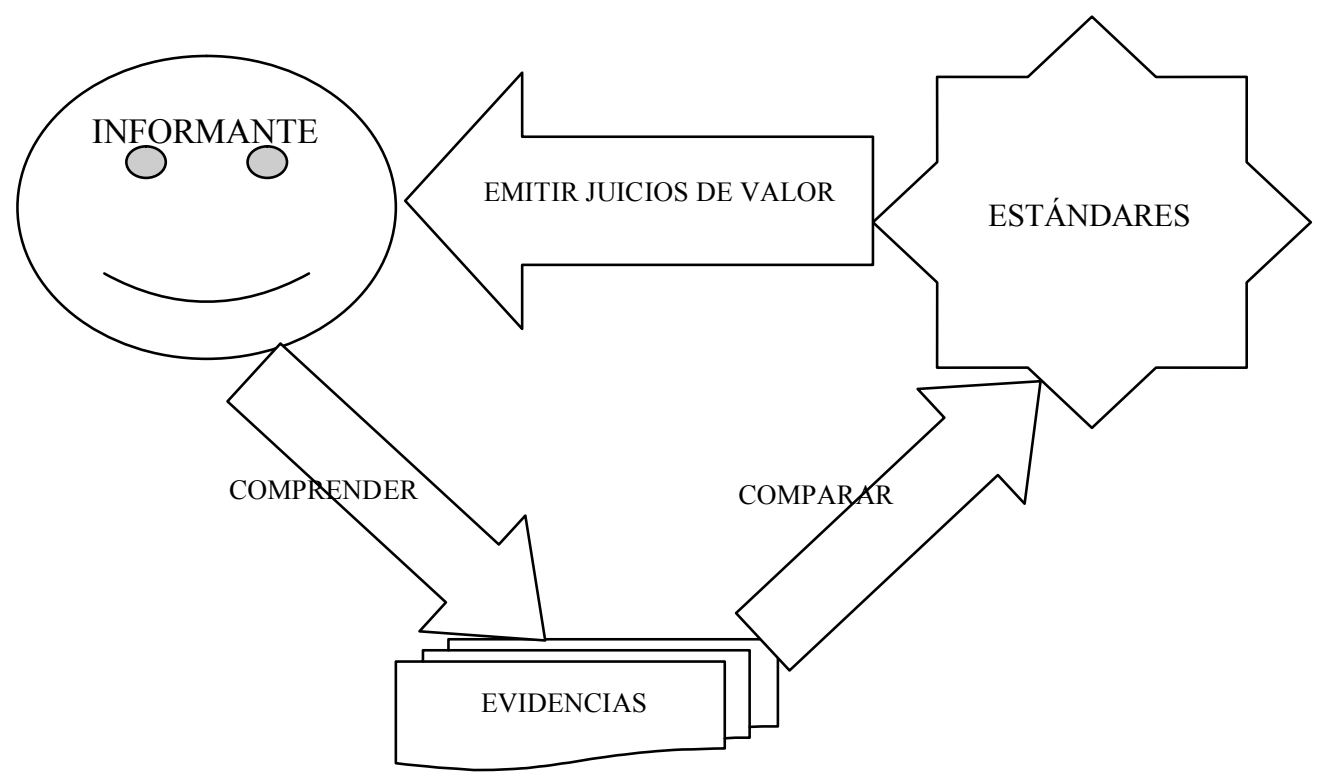

Figura 1. Esquema del proceso individual utilizado por los funcionarios de la UTEQ para autoevaluar cada uno de los indicadores que le correspondía. 
gestores universitarios está aportando a la construcción de la calidad institucional. Estas actividades generaron expectativas e interés, ya que cada uno de los consultados podía opinar libremente sobre el tema y sustentar su opinión en los referentes para evaluar, y la información disponible sobre el indicador. Cada uno de los Comités de las Unidades Académicas se responsabilizó de la capacitación y presentación de la información pertinente a los actores universitarios que serían consultados. En esta etapa también los funcionarios universitarios, hicieron su análisis de fortalezas y debilidades encontradas en cada uno de los indicadores a su cargo. Interpretaron los datos y hechos para generar información y determinaron el grado de cumplimiento del indicador en una escala de 0 a 100.

\section{El Informe Documentado}

$\mathrm{P}$ ra que todos los funcionarios universitarios identificados como responsables del cumplimiento de los indicadores a su cargo, emitan sus juicios de valor sustentados en las evidencias que disponían, (Transforman la Información en Conocimiento) se les solicitó elaboren un informe documentado, con la estructura que se indica a continuación.

\section{Identificación del indicador, evidencias y referente para evaluar}

Aquí se adjunta la Matriz de Evaluación construida por la CEI, para todos los indicadores, utilizando los estándares y preguntas orientadoras descritas en el Manual de Evaluación Externa (CONEA, 2005) y la Matriz de Ponderación de la Guía de autoevaluación institucional del CONEA.

La matriz permite visualizar la relación que existió entre el indicador, las evidencias, el referente para evaluar y el grado de cumplimiento (Cuadro 1).

Cuadro 1. Ejemplo de matriz de evaluación para un indicador (CEI, 2008)

\begin{tabular}{|c|c|c|c|}
\hline Código & Indicador & Evidencias & Referente para autoevaluar \\
\hline \multirow[t]{7}{*}{ RA.RM.2.5.05 } & Listado de: & Listado de: & La carrera dispone de: \\
\hline & $\begin{array}{l}\text { - Áreas y campos } \\
\text { experimentales } \\
\text { equipados }\end{array}$ & $\begin{array}{l}\text { Áreas y campos experimentales, } \\
\text { proyectos didácticos productivos } \\
\text { equipados por carrera }\end{array}$ & $\begin{array}{l}\text { Áreas y campos experimentales, pro- } \\
\text { yectos didácticos productivos }\end{array}$ \\
\hline & & & $\begin{array}{l}\text { Equipados y funcionales destinadas a } \\
\text { prácticas y experimentación por carre- } \\
\text { ra. }\end{array}$ \\
\hline & $\begin{array}{l}\text { - Y funcionales por } \\
\text { carrera }\end{array}$ & & \\
\hline & & $\begin{array}{l}\text { Llenar la Guía de Observación } \\
\text { para cada uno de los menciona- } \\
\text { dos }\end{array}$ & \\
\hline & & & $\begin{array}{l}\text { La dotación de los espacios es sufi- } \\
\text { ciente para atender la demanda de los } \\
\text { estudiantes }\end{array}$ \\
\hline & & & $\begin{array}{l}\text { El estado de cada una de los campos } \\
\text { es funcional y apropiado. }\end{array}$ \\
\hline
\end{tabular}

\section{Análisis FODA.}

En este acápite se describió un análisis concreto de las evidencias disponibles. Es necesario interpretar los hechos, datos, o información que la universidad tiene como evidencia del indicador en análisis, a fin de transformarla, resumirla o generar nueva información clara, objetiva, precisa y pertinente

\section{Argumentos que sustentan el grado de cumplimien- to}

Aquí se señalaron las explicaciones que sustentan la valoración asignada por el informante. Fueron la consecuencia del análisis comparativo entre la información disponible (lo que tenemos) y el estándar o referente para evaluar (lo que deberíamos tener). Traducen la información en conocimiento y dependen en gran medi- 
da del referente para autoevaluar. Se demostró también el grado de compromiso y auto exigencia del informante con la calidad universitaria. Estos argumentos fueron la base para formular planes de mejora y avanzar en la construcción de la calidad universitaria. Evidenciaron los valores y principios del informante con relación a la calidad institucional, su desempeño y contribución al éxito de la universidad para alcanzar la acreditación.

\section{Juicios de valor y valoración del indicador}

Se registraron los juicios de valores cualitativos y cuantitativos, es decir, el grado de cumplimiento del indicador, derivados del análisis y comparación de las evidencias con el referente para evaluar y el estándar respectivo. Se utilizó la escala propuesta por el CONEA para los juicios cualitativos y una escala de 0 a 100 para los juicios cuantitativos.

\section{Codificación de evidencias presentadas}

Los códigos permitieron reunir las evidencias presentadas por los informantes individuales, cuando el indicador tuvo más de un informante, en un archivo global de la universidad, organizado por estándares, indicadores y referentes para evaluar. El archivo así organizado puede ser revisado con mayor facilidad por los evaluadores externos.

\section{Acciones o planes de mejora propuestos}

Utilizando el manual publicado por el Comité Técnico de la CEI, para elaborar planes de mejora con la metodología del marco lógico, y las recomendaciones del CONEA, para cada uno de los indicadores identificados como insuficientes para acreditar (calificación menor a $76 \%$ ) es decir, fortalezas tipo B, el informante elaboró y presentó planes de mejora.

Con el proceso descrito, se trató de demostrar que los funcionarios elegidos como informantes calificados exhibían competencias indagativas, argumentativas e innovativas. En la (Figura 2), se resumen los procedimientos seguidos:

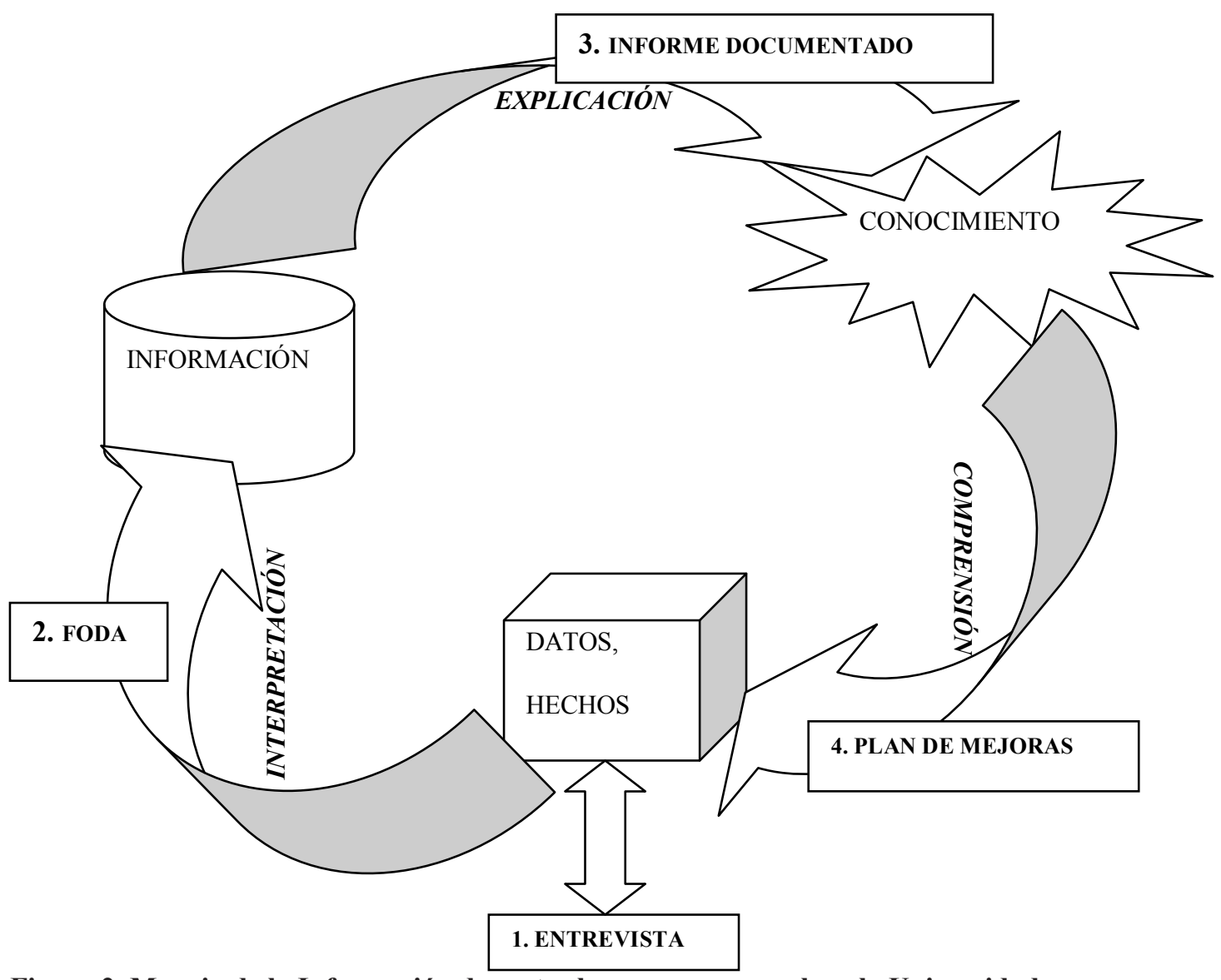

Figura 2. Manejo de la Información durante el proceso para evaluar la Universidad 
La tercera etapa, consistió en la recopilación de los juicios de valor mediante un cuestionario auto administrado y contestado por la mayoría de los informantes calificados del medio interno mediante un censo, utilizando el sitio de la CEI en la página www.uteq. edu.ec. Los juicios de valor se expresaron en cantidades entre 0 y 100 donde el intervalo 0 - 25 correspondía a la Categoría Insuficiente; de 26 - 50 Regular; entre 51 - 75 Buena y si el informante asignaba una calificación entre 76 y 100 significaba que su juicio de valor estaba en la Categoría Muy Buena

La tercera etapa (De la Información al Conocimiento) concluyó para los funcionarios universitarios con la propuesta de acciones, planes o proyectos de mejoras, algunos funcionarios, inmediatamente iniciaron acciones de mejoras para cumplir con el indicador.
Otros propusieron planes de mejoras detallados.

En la mayoría de casos, hicieron propuestas de acciones de mejoras que posteriormente fueron compiladas en talleres para elaborar planes de mejoras institucionales para los ocho ámbitos descritos en la guía de autoevaluación (CONEA, 2003b). Estos planes fueron incorporados al PEDI Institucional UTEQ 2008 - 2012 en vigencia.

\section{Resultados con la metodología utilizada}

La participación de los informantes calificados en el medio interno y externo se puede notar en el Cuadro 2:

Cuadro 2. Evolución del número de indicadores verificados con debilidades (CEI, 2008)

\begin{tabular}{lccc}
\hline \multicolumn{1}{c}{ Función } & $\begin{array}{c}\text { Antes } \\
\text { autoevaluación }\end{array}$ & $\begin{array}{c}\text { Después de la } \\
\text { autoevaluación }\end{array}$ & $\begin{array}{c}\text { Con la evaluación } \\
\text { externa }\end{array}$ \\
\hline Gestión administrativa & 34 & 3 & 7 \\
Docencia & 47 & 6 & 0 \\
Investigación & 11 & 2 & 1 \\
Vinculación con la Colectividad & 8 & 0 & 0 \\
\hline TOTAL & $\mathbf{1 0 0}$ & $\mathbf{1 1}$ & $\mathbf{8}$ \\
\hline
\end{tabular}

Se logró que participaran casi todos los actores universitarios del medio interno y de una gran proporción de informantes del medio externo quienes fueron motivados por los comentarios positivos que surgieron especialmente de los alumnos, quienes expresaron su satisfacción por tener un medio para expresar sus opiniones, las cuales además, fueron tomadas en cuenta y sirvieron para formular planes de mejora que iniciaron procesos de cambio, muchos de los cuales eran esperados por la comunidad universitaria.

En el diseño del Proyecto de Autoevaluación se identificaron indicadores que aparentemente no contaban con evidencias. La CEI, al inicio del proceso, elaboró un resumen de los indicadores que no superaban la ponderación mínima para acreditar, informe que fue presentado al HCU. Durante el proceso, las fallas se fueron corrigiendo hasta alcanzar los requisitos exigidos por los estándares del CONEA. Finalmente, con la evaluación externa se confirmó si los cambios fueron suficientes para acreditar.

Para la mayoría de los indicadores, las evidencias no estaban disponibles. Muchos indicadores defini- tivamente no contaban con evidencias a pesar de existir información de actividades desarrolladas y para algunos indicadores, no existían ni actividades desarrolladas, ni evidencias.

En el Cuadro 2 se presenta la evolución del número de indicadores verificados como debilidades, con calificación menor a 76\% (no acreditan) antes de la evaluación, con la autoevaluación y con la evaluación externa.

El Comité de Evaluación Externa confirmó las calificaciones de 166 de los 180 indicadores utilizados para recolectar la información, a 9 indicadores les asignó una calificación menor (aun así dos acreditaron) y a los 5 indicadores restantes les asignó una mayor calificación a la asignada por los funcionarios universitarios calificados como informantes del medio interno (Capa et al., 2008). Es decir que el 5\% de los indicadores fueron corregidos con calificación menor y un $2.7 \%$ de los indicadores mejoraron su calificación. Por lo expuesto, se puede decir que la autoevaluación y la evaluación externa llegaron a conclusiones similares, en la mayoría de los casos. 
Cuadro 3. Instrumentos y número de participantes del proceso (CEI, 2008)

\begin{tabular}{lc}
\hline Descripción & Participantes \\
\hline Cuestionarios vía Internet & 4,067 \\
Entrevistas personales & 41 \\
Guías de observación & 40 \\
Entrevistas a Empresarios & 117 \\
Encuestas Actores externos beneficiarios del DBU & 12 \\
Encuestas Actores externos beneficiarios del DEVITTEC & 15 \\
Encuestas Egresados y Graduados que laboran en el medio externo & 132 \\
Encuestas Egresados y Graduados que laboran en la UTEQ & 44 \\
Estudiantes en Talleres & 300 \\
\hline Total Informantes & $\mathbf{4 , 7 6 8}$ \\
\hline
\end{tabular}

Como consecuencias del proceso, el Informe de Autoevaluación (CEI, 2008) señala:

- La comunidad universitaria se ha empoderado de la cultura de evaluación.

- La universidad está más organizada en su accionar, interesada en tener evidencias de todo el trabajo universitario

-Implementación de un Modelo Pedagógico por Competencias

-Elaboración del nuevo Plan Estratégico de Desarrollo

Integral, para los próximos 5 años (2008-2012)

-Elaboración de un nuevo Orgánico Funcional

-Reformulación del Estatuto

- Reformular el presupuesto 2008 para cumplir planes de mejoras derivados del proceso de autoevaluación

Por su parte el informe de Evaluación Externa (Capa et al., 2008) en lo pertinente, también señala que:

- El nivel de información con respecto al proceso de evaluación fue alto entre la comunidad universitaria - La información utilizada en el proceso de autoevaluación ha sido bastante completa, con base en documentos generados por la universidad o encuestas a la comunidad universitaria y a informantes del sector externo; por lo cual, ciertas debilidades han sido claramente señaladas e incorporadas en el plan de mejoras.

- El diagnóstico contenido en el informe de autoevaluación ha sido realizado con información completa, pero además su veracidad ha sido constatada con la entrega de documentos solicitados a las diferentes unidades de la UTEQ.

-En general, se presentan proyectos bien elaborados para dar cumplimiento al plan de mejoras.

- La elaboración de proyectos para dar cumplimiento al plan de mejoras han sido desarrollados con bastante profundidad y su ejecución, en general, marcha de manera normal.

-Se ha interiorizado la necesidad de sistematizar el mo- nitoreo de ciertos aspectos que aparecen como fortalezas, pero que por el momento no son adecuadamente medidos, como son las actividades vinculadas con la colectividad.

Cada informante es un evaluador de la calidad universitaria desde su perspectiva, con su conocimiento e información, con su propósito, pero con el mismo referente o criterio para comparar y emitir juicios de valor. Por lo tanto, está en posición de hacer propuestas de mejoras que además de contribuir con la calidad universitaria, a él le interesa cumplir o le gustaría cumplir y así aportar para mejorar la calidad universitaria, que es el propósito primordial de este proceso. Si no es él quien ha llegado a conclusiones y juicios de valor que guíen su acción, no habrá compromiso con las mejoras.

Por ejemplo, consultar sobre el cumplimiento de principios y valores de cada uno de los estudiantes, docentes, o empleados, dentro de los predios universitarios, con la posibilidad de que esa opinión pueda ser verificada por evaluadores externos imparciales, compromete a cada informante, ya que no es una opinión sin consecuencias. Se trata de un compromiso que debe ser cumplido en el futuro, especialmente si existe la posibilidad de obtener la acreditación que incrementará el prestigio universitario y en consecuencia el respaldo al título obtenido al finalizar los estudios o al reconocimiento social que significa trabajar en una institución acreditada.

Los planes de mejoras una vez aplicados deberían modificar la situación inicial, y originar nuevos datos y hechos que sustentarían un nuevo ciclo de evaluación.

En el caso que nos ocupa, la calidad de los planes de mejoras estuvo muy relacionada con el conocimiento generado por cada informante para cada uno de los problemas que detectó. Pero la profundidad y la duración de los cambios iniciados con el proceso de Autoevaluación, deben ser estudiadas posteriormente 
y más parece ser que dependen de los procesos Administrativos y Motivacionales utilizados para aplicar los planes de mejoras propuestos.

Si convenimos que la calidad universitaria es la consecuencia del trabajo individual y colectivo de cada uno de los involucrados en los procesos educativos, administrativos, investigativos y de vinculación con la colectividad, entonces la autoevaluación institucional es una oportunidad para mejorar el desempeño de cada uno de los actores universitarios: Autoridades, Directivos, Docentes, Empleados, Trabajadores, Estudiantes e incluso graduados, beneficiarios, proveedores, colaboradores, examinadores y otros actores vinculados con la UTEQ.

\section{Conclusiones}

$\mathrm{L}^{2}$ autoevaluación institucional es un trabajo complejo y delicado que requiere una metodología muy detallada y la participación conjunta de todos los actores, quienes deben comprender el propósito, los criterios y el procedimiento para analizar hechos, datos o información y emitir su juicio de valor con base en las evidencias.

La autoevaluación desarrollada con el proceso diseñado por la UTEQ, arrojó resultados similares a los de la evaluación externa y generó actitudes positivas al cambio dentro de la comunidad universitaria, pero la duración y profundidad de los cambios iniciados debe ser valorada después de un tiempo prudencial.

\section{Literatura CITADA}

Capa, H., W. Medina, B. Pástor y O. Zorrilla. 2008. Evaluación Externa Universidad Técnica Estatal de Quevedo. Quito: Consejo Nacional de Evaluación y Acreditación CONEA, 2008.
CEI (Comisión de Evaluación Institucional). 2006. Proyecto de Autoevaluación Institucional con fines de acreditación. Quevedo Ecuador : Editorial Universitaria UTEQ, 2006.

CEI (Comisión de Autoevaluación Institucional). 2008. Informe Final de Autoevaluación. Quevedo, Ecuador: Editorial Universitaria, UTEQ. 2008.

CONEA (Consejo Nacional de Evaluación y Acreditación de la Educación Superior del Ecuador). 2003a. La Calidad en la Universidad Ecuatoriana. Quito, Ecuador : Registro Oficial No 218, 2003.

CONEA (Consejo Nacional de Evaluación y Acreditación de la Educación Superior del Ecuador). 2003 b. Guía de Autoevaluación con fines de Acreditación para las Universidades y Escuelas Politécnicas del Ecuador. serie Documentos Técnicos, N $\mathrm{N}^{\mathrm{O}} 3$. Quito Ecuador : Registro Oficial No 218, 2003.

CONEA (Consejo Nacional de Evaluación y Acreditación de la Educación Superior del Ecuador). 2005. Manual de Evaluación Externa Institucional para la Acreditación de Universidades y Escuelas Politécnicas. Quito, Ecuador : Registro Oficial 4 enero 2005, 2005. No 496.

Soria, R. 2008. Procedimiento para Autoevaluar en la UTEQ. Universidad Técnica Estatal de Quevedo. [En línea] 24 de Abril de 2008. [Citado el: 24 de Abril de 2008.] www.uteq.edu.ec.

UP (Unidad de Posgrado). 2008. Diseño y Ejecución de Proyectos de Evaluación del Aprendizaje Social Cognitivo. Quevedo, Ecuador : Asociación Docentes UTEQ, 2008. Diplomado Superior en Diseños Pedagógicos Universitarios, Modulo III. págs. Guía de Autoestudio, Actividad Grupal. 